\title{
Una propuesta empírica de creación de cuadro de mando para pyme desde una interpretación estratégica de las normas internacionales de información financiera
}

\author{
Santiago García Carvajal*, Robinson Dueñas**, Henry Acuña Barrantes***
}

* Maestría en Administración de Empresas. Docente investigador, Universidad La Gran Colombia, Bogotá, Colombia. Correo electrónico: santiago.garcia@ugc.edu.co

* Magíster en Relaciones Internacionales. Docente investigador, Universidad Militar Nueva Granada Bogotá, Colombia. Correo electrónico: robinsonduenasc@gmail.com

*** Doctorando en Negocios Internacionales, Escuela Española de Negocios Internacionales, Tarragona, España. Correo electrónico: hacuna@reingex.com

Recibido: 22 de julio del 2016

Aprobado: 7 de noviembre del 2016

Cómo citar este artículo: García-Carvajal, S. Dueñas, R. y Acuña-Barrantes, H. (2017). Una propuesta empírica de creación de cuadro de mando para Pyme desde una interpretación estratégica de las normas internacionales de información financiera. Cooperativismo \& Desarrollo, 110(25), 37-53. doi: https://doi. org/10.16925/co.v25i110.1759.

\section{Resumen}

Introducción: hoy en día, surge la necesidad de crear y diseñar procedimientos que tienen como fin el funcionamiento del sistema empresarial en el ámbito universal. Dentro de estos avances, pueden mencionarse las normas internacionales de información financiera, siendo estas las más discutidas y de considerable aplicación en todo el ámbito empresarial internacional. Metodología: se plantea una revisión de los procesos de convergencia de las normas estándares de contabilidad a las normas internacionales de información financiera, de tal forma que interprete las posibles consecuencias que podrían darse en la empresa en todas las disciplinas organizacionales sobre la plataforma de un cuadro de mando operativo; esto último corroborado por un análisis correlacional estadístico realizado a pymes colombianas en SPss. Resultados: en la elaboración del trabajo, se han analizado los procedimientos de la NIIF para las PYME adoptando dicha norma para preparar estados financieros en un cuadro de mando, dicho cuadro es correlacional en la implementación del modelo en las pymes, los resultados fueron más que probabilísticos. Conclusiones: lo anterior lleva a la conclusión de que es una herramienta de gran utilidad para cualquier compañía; el cambio contable que la adopción de la norma implica contempla criterios simplificados.

Palabras clave: balanced score card, mercados emergentes, normas internacionales de información financiera, pymes. 


\title{
Empirical Proposal to Create a Scorecard for sMEs from a Strategic Interpretation of the International Financial Reporting Standards
}

\begin{abstract}
Introduction: Nowadays the need to create and design procedures that have as purpose the adequate functioning of the business system in a universal scale arises. These advances include the IFRS, International Financial Reporting Standards, which are the most discussed and which present considerable application cases throughout the whole international business arena. Methodology: A review of the convergence processes of the traditional accounting standards to the IFRS is proposed, so as to interpret the possible consequences that could emerge in all the organizational disciplines inside the company, mounted on the platform of an operational scorecard and corroborated by a correlational statistical analysis done to Colombian sMes using sPss. Results: To elaborate this study, the IFRS procedures for SMES were analyzed, adopting IFRS to prepare financial statements in a scorecard, which is correlational in the implementation of the model for SMEs. The results were more than probabilistic. Conclusions: IFRs constitute a tool of great utility for any company, the adoption of IFRS implies en accounting change that simplifies criteria.
\end{abstract}

Keywords: balanced dashboard, emerging markets, international financial reporting standards (IFRS), SMES.

\section{Uma proposta empírica de criação de painel de gestão para PME a partir de uma interpretação estratégica das normas internacionais de informação financeira}

\section{Resumo}

Introdução: hoje em dia, surge a necessidade de criar e desenhar procedimentos que têm como objetivo o funcionamento do sistema empresarial no âmbito universal. Dentro desses avanços, podem ser mencionadas as normas internacionais de informação financeira, sendo estas as mais discutidas e de considerável aplicação em todo o contexto empresarial internacional. Metodologia: propõe-se uma revisão dos processos de convergência das normas-padrão de contabilidade às normas internacionais de informação financeira, de tal forma que interprete as possíveis consequências que poderiam ocorrer na empresa em todas as disciplinas organizacionais sobre a plataforma de um painel de gestão operativo, este último corroborado por uma análise correlacional estatística realizada a PMES colombianas em sPss. Resultados: na elaboração do trabalho, foram analisados os procedimentos da NIIF para as PMEs adotando essa norma para preparar estados financeiros num painel de gestão; esse painel é correlacional na implantação do modelo das pMes; os resultados foram mais probabilísticos. Conclusões: o anterior leva à conclusão de que é uma ferramenta de grande utilidade para qualquer companhia; a mudança contábil que a adoção da norma implica contempla critérios simplificados.

Palavras-chave: balanced score card, mercados emergentes, normas internacionais de informação financeira, PMEs. 


\section{Introducción $^{1}$}

Se utilizarán como indicadores las 35 secciones de las Normas Internacionales de Información Financiera (NIIF) para PYME como modelo econométrico de análisis de desempeño por categorías. Al momento de implementar las NIIF, se debe tener en cuenta qué secciones se adoptan según las necesidades del cuadro de mando, pues siempre que existen cambios estos generan resistencia, para lo cual se determina realizar capacitaciones y generar liderazgo activo, abordando el tema de PYME hacia un patrón empresarial competitivo.

El concepto de globalización, derivado de las disciplinas sociales internacionales, ha influenciado las políticas de internacionalización de la empresa, las teorías de la competitividad y las nuevas teorías del comercio internacional. Hoy es imperativa la necesidad de estandarizar los procesos de auditoría y control de gestión para homologar los procesos contables de distintos países, de manera que facilite la penetración de mercados multinacionales, la inversión extranjera y los procesos de auditoría internacional. El artículo de investigación plantea la importancia y el desarrollo de una política de internacionalización de las PYME colombianas que propenda a la eficiencia y la certeza en el logro de objetivos organizacionales; sin embargo, para la gerencia el paradigma de la complejidad siempre está presente. La complejidad es un enfoque que considera a la organización como un espacio en el que coexisten el orden y el desorden, la razón y la sin razón, las armonías y disonancias; por eso, este proyecto se propone crear en las PYME un cuadro de mando estratégico integrado en las Normas Internacionales de Información Financiera (NIIF) que dé importancia tanto a los sentidos comunes como al sentido emergente de las interacciones entre grupos, los intercambios en un ambiente incierto y cambiante, con una competencia agresiva, en el cual la innovación tecnológica lleva al acortamiento de los ciclos de renovación tanto en métodos y equipos de producción como en bienes y servicios finales.

Es interesante observar los estudios que ha realizado el Banco Mundial (2002), en los cuales enuncian que:

Artículo de Investigación, derivado del proyecto de investigación DIS-1812, titulado "Cuadro de mando integrado NIIF para pymes”, financiado por la Universidad La Gran Colombia y la Universidad Militar Nueva Granada.
Esto se evidencia con experiencias internacionales como en el caso de Taiwán, donde el 98\% de las empresas son mi PYME, las cuales emplean al $70 \%$ de la población activa y aportan el $65 \%$ del volumen total de las exportaciones. En otros países como Chile las mi PYME representan el $92 \%$ del parque empresarial con una participación del 40,8\% en las exportaciones.

En el caso de Colombia, donde hay grandes limitaciones de información en este campo, se estima que las empresas de menor tamaño agrupan un $91,3 \%$ solo en el sector manufacturero; estas generan cerca del $42 \%$ del empleo industrial y realizan un poco más del $20 \%$ de las exportaciones industriales (DANE, 2001). Concretamente, al mirar la evolución de las PYME en Colombia, se observa cómo, a pesar de las políticas de estímulo a esta clase de empresas, el promedio de antigüedad de las PYME en Colombia es de 10 años

Las Normas Internacionales de Información Financiera (IFRS, por sus siglas en inglés) surgieron de las dificultades de comparar los informes financieros entre un país y otro, dado que cada uno tenía sus propias normas contables. Bajo este esquema, se hace complejo tomar decisiones de negocio en distintos países al no contar con información homogénea. Están diseñadas para ser aplicadas en los estados financieros con propósito general, de entidades con ánimo de lucro, estableciendo requisitos para el reconocimiento, baja en cuenta, medición, presentación y revelación. Aplican tanto a compañías individuales como estados financieros consolidados (Viveros, 2014).

El cuadro de mando estratégico fue creado por Robert Kaplan y David Norton en 1992; este fue aplicado primordialmente a empresas con ánimo de lucro en Europa y Estados Unidos. Este funciona como una consola de mando en un carro de carreras usado por los pilotos para supervisar las condiciones del carro y al mismo tiempo respondiendo estratégicamente a los movimientos de otros carros en la pista de carreras. En este caso el propósito es adoptar una estructura empresarial más sólida y aplicar herramientas gerenciales más estructuradas que faciliten el desempeño organizacional y, al mismo tiempo, les permitan aumentar su nivel de productividad.

El cuadro de mando estratégico es un sistema integrado porque emplea cada una de las perspectivas del negocio, las cuales son indispensables para visualizar la firma como un todo. Es un sistema balanceado debido a que es esencial que la estrategia sea coherente y balanceada con el grupo de indicadores ya sean estos financieros o sean obtenidos como un resultado 
del proceso. Es estratégico ya que los objetivos están relacionados de tal forma que la estrategia se traduce en un mapa de causa y efecto.

El propósito principal de la herramienta del cuadro de mando estratégico no es simplemente el de desarrollar un grupo de indicadores, a pesar de que esos indicadores ayudan a describir los objetivos y las metas del proyecto, los resultados proveen el enlace que evalúa el personal para el alcance de una estrategia exitosa. El uso potencial de una estructura de indicadores debe perseguir la creación de un sistema integrado gerencial. Kaplan y Norton (1996) indicaron que "las estructuras basadas en indicadores no deberían solamente buscar los medios para obtener una meta de mayor alcance: un sistema estratégico gerencial que ayude a ejecutivos a implementar un instrumento para obtener retroalimentación del desempeño de la estrategia” (p. 283).

\section{Revisión literaria}

Según estudios de Muñoz (2009), las NIIf para PYME:

Son un conjunto autocontenido de principios de contabilidad que se basan en los IFrs plenos, pero que ha sido simplificado para que sea adecuado para las PYME. Por ello, el estándar ha sido organizado por temas para hacer que se parezca a un manual de referencia. [La Junta de Normas Internacionales de Contabilidad] IASB tiene la intención de que sea más amigable para los preparadores de las PYME y para los usuarios de los estados financieros de las PYME.

Entre otras características, las PYME se reconocen como organizaciones que realizan transacciones de pequeño o mediano volumen. Al igual que sus operaciones monetarias, la cantidad de personas que trabajan en ella no son definitivas e iguales para todas y pueden diferir entre un país industrializado y un país en desarrollo; es por esto que no es posible definirlas solo en términos cuantitativos, es necesario también establecer las cualidades del negocio.

La Declaración Internacional sobre Prácticas de Auditoría (IAPS) 1005 le asigna a estas entidades las siguientes características:

1. La concentración de la propiedad y administración pertenece a un número pequeño de individuos (generalmente un solo individuo).

2. Tienen una o más delas siguientes particularidades:

a. Pocas fuentes de ingresos.

b. Sistemas de registros no sofisticados. c. Controles internos limitados y la posibilidad de que la administración sobrepase los controles.

Este tipo de empresas no presentan necesariamente todas las características mencionadas anteriormente, pero se clasificarán aquí solo aquellas que posean alguna de las particularidades siguientes.

La aplicación de controles internos sofisticados en estas empresas puede resultar innecesario. Las funciones se concentran en pocas personas y el propietario administrador es quien ejerce algunos controles. Entonces, para asegurar la calidad y validez de los procesos, se hace necesaria la segregación de funciones en cargos clave y la implementación de controles por parte del propietario administrador que propendan a la salvaguarda de los activos a través de la medición del desempeño y el control de las operaciones.

En este tipo de empresas, las decisiones y el direccionamiento de la entidad corren por cuenta del propietario administrador. Cuando las funciones se encuentran demasiado concentradas se requiere, para resguardarse de fugas de dineros, que el propietario administrador autorice todas las transacciones relacionadas con compras y pagos, de tal manera que exista un control severo de estas actividades para detectar intenciones de fraude o errores cometidos.

Los poderes asignados al administrador, cuando no es el propietario, pueden conllevar a la ejecución de fraudes por parte de este. La auditoría que se ejerza deberá ser muy estricta al momento de evaluar la administración y deberá investigar y verificar las operaciones que pudieren ser influidas por el administrador y que indiquen la presencia de malversaciones.

Al investigar profundamente las actividades administrativas, no se trata de establecer la deshonestidad del administrador. El auditor, haciendo uso de su escepticismo profesional, debe expresar una opinión objetiva sobre los manejos de la administración sin plantear juicios incriminatorios ni respaldar su absoluta honradez (Salazar, 2013).

Además de las NIIF plenas, ha desarrollado y elaborado NIIF para PYME (IFRS por sus siglas en inglés). Estos estándares aplican a aquellas entidades privadas y entidades sin obligación pública de rendir cuentas.

Para el Consejo de Normas Internacionales de Contabilidad (2009):

Las NIIF están diseñadas para ser aplicadas en los estados financieros con propósito de información general, así como en otra información financiera, de todas las entidades con ánimo de lucro. Los estados 
financieros con propósito de información general se dirigen a la satisfacción de las necesidades comunes de información de un amplio espectro de usuarios, por ejemplo, accionistas, acreedores, empleados y público en general. El objetivo de los estados financieros es suministrar información sobre la situación financiera, el rendimiento y los flujos de efectivo de una entidad, que sea útil para esos usuarios al tomar decisiones económicas (p. 11).

A su vez, un patrón estratégico que se debe seguir son los cuadros de mando integral (CMI), los cuales se diferencian de otras herramientas de manejos de negocios en que están más orientados al seguimiento de indicadores que al análisis minucioso de información.

Por otro lado, es muy común que un CMI sea controlado por la dirección general de la empresa. Por tanto, los gerentes analizan el mercado y la estrategia para construir un modelo de negocio que refleje las interrelaciones entre los diferentes componentes de la empresa (o llamado también plan estratégico). Una vez que lo han construido, los responsables de la PYME lo utilizan como modelo.

$\mathrm{Al}$ trazar CMI en un plan estratégico cuantitativo, este implanta las cifras que debe alcanzar la pyme; este describe el modo de conseguirlas, perfilando la estrategia a seguir, indicando los plazos de los que dispone la empresa para alcanzar esas cifras.

Para lograrlo, se requiere ajustarlo a las necesidades de cada pyme y se debe incorporar en la reflexión al particular de la empresa. Cuanto mayor sea la participación de las personas y áreas (de ahí la importancia de lo trabajado anteriormente) que integran la empresa, mayor será su compromiso y, por tanto, se tendrá más posibilidades de tener un plan estratégico con éxito.

\section{Metodología}

La metodología de esta investigación está orientada a realizar unos procesos de convergencia que se articulen con los planes gubernamentales contemplados en la normatividad vigente, por ejemplo, el artículo 7 de la Ley 1314 de 2009, sobre las bases técnicas de aceptación universal, comparables y con credibilidad, que contemplen las variables que afectan el desarrollo contable en relación entre los negocios y los proveedores de capital.

A diferencia de los países anglosajones, los cuales están más orientados a la predicción con sus sistemas contables, dirigidos a inversores y el mercado, cuyos objetivos son evaluar la situación presente y futura de la empresa, y propenden a la separación entre contabilidad-fiscalidad y protección del mercado, los países hispanos están orientados al control: reportan su información dirigida a propietarios y acreedores, su objetivo es la rendición de cuentas y control, mantienen influencia fiscal y se enfocan en la protección patrimonial.

Entorno y ámbito de aplicación de las NIIF y de las Normas Internacionales de Contabilidad (NIC): contextualizar de forma global el marco de aplicación NIIF y establecer un cuadro de mando que permita guiar a los responsables de los informes de gestión y de la planeación financiera.

Los estándares internacionales de auditoría (ISA), en su concepción inicial, fueron diseñados para su aplicación en cualquier tipo de organización sin interesar su tamaño, la naturaleza jurídica, la forma de administración, ni el tipo de operaciones.

Los estándares internacionales de auditoría, aseguramiento y servicio relacionados son emitidos por el Comité Internacional de Normas de Auditoría y Aseguramiento (IAASB), el cual reemplazó en abril del 2002 al Comité de Prácticas de Auditoría (IAPAC), el cual venía funcionando desde 1997.

El IAAsB depende de la Federación internacional de Contadores (IFAC) y tiene como finalidad:

1. Servir al interés público fiando estándares de auditoría y aseguramiento de alta calidad.

2. Proveer la convergencia de las normas nacionales con los estándares internacionales de auditoría, para así lograr la uniformidad en la práctica de la auditoría alrededor del mundo.

3. Fortalecer la confianza en la auditoría en el mundo.

El IAASB trabajó desde el 2003 en el proyecto Claridad, el cual se terminó a finales del 2009. Este proyecto buscó los siguientes objetivos:

1. Uniformar la estructura en la redacción de cada ISA, fijando los objetivos de cada una y las obligaciones del auditor.

2. Mejorar su comprensibilidad.

3. Unificar ciertos términos, enfatizando el carácter obligatorio de su cumplimiento.

4. Eliminar la redacción ambigua.

5. Introducción de cambios sustanciales mediante un proceso de revisión a prácticamente el $50 \%$ de las ISA. 
6. Definir una misma fecha de aplicación de las ISA.

Características de las pequeñas y medianas empresas: la PYME generalmente se maneja con documentación y tecnología menos compleja a la utilizada en las grandes empresas. Es por lo anterior que los cambios que genera la aplicación de las NIIF dentro de una organización no solo afectan al área contable-financiera, sino a todas las áreas, procesos y sistemas de la empresa, es decir que el aprendizaje debe integrar a todo personal implicado con las operaciones y financiamiento de la organización.

Por tal motivo, se considera que se debe implementar un proceso de capacitación sobre las NIIF con el fin de que estas permeen en la estructura organizacional, logrando así los objetivos empresariales adecuándolos a las normas internacionales.

\section{Procedimiento}

La persona responsable del proyecto es quien debe liderar el proyecto (gerente) y realizar un plan de implementación en el cual se realice la capacitación de los funcionares, incluido el líder, según el cargo que desempeñen.

Por consiguiente, se debería capacitar a:

1. Gerentes (administrador): esta persona es la encargada de dirigir la organización y tomar las decisiones más apropiadas teniendo siempre en cuenta el factor humano, ya que las personas son el factor primordial en cualquier organización: brindarles la capacitación, los descansos y lo necesario para que se sientan augustos y cómodos con el trabajo que conlleva la implementación de las NIIF.

Estará al tanto de la rentabilidad y la capacidad financiera, ya que la financiación del desarollo es como invierto el capital que poseo. Como bien se sabe, la implementación de las NiIf genera costos, sobre los cuales el administrador deberá estar al tanto para no invertir en estas normas más del capital que se posee, deberá adecuarse a todos los cambios que se generen en el entorno y así anticiparse a los problemas que pueden darse.

Las habilidades que deben poseer los administradores son las siguientes: adaptación al aplicar el conocimiento contable para resolver problemas del mundo real, dejando a un lado las prácticas tradicionales; comunicación tanto en la organización como con personas de otras culturas y transferir conocimiento; manejo segundo idioma (inglés); negociación en ambientes globales y otras culturas; capacidades intelectuales de investigación, de encontrar soluciones, de pensamiento lógico y razonable, entre otros; habilidades interpersonales para el trabajo en equipo, resolver conflictos y motivar al personal; ser un visionario con conducta ética, ser creativo, persistente, etc., y tener un compromiso de aprendizaje para toda la vida.

2. Contadores: es el contador quien lleva el procedimiento a cabo. Es el encargado de llevar los hechos y transacciones económicos dentro de la organización, cumpliendo así las normas y reglas propuestas para este fin. Las nuevas normas internacionales exigen, por lo tanto, a todos los profesionales, conocer, estudiar, determinar su contenido, impacto, sus procesos y procedimientos para la manipulación de la información

El papel de los contadores es fundamental ya que deben contar con las siguientes competencias: interpretar la norma y examinar cómo la aplica a una empresa, dependiendo el sector productivo que esta maneje; analizar esos datos registrados emitiendo informes mucho más profundos y detallados sobre la realidad financiera y económica de la empresa, y dominar técnicas cuantitativas de áreas como la econometría, la informática, el pronóstico, la gerencia y la dirección, entre otros.

3. Auditores: los auditores deben conocer las NIIF. Debido a que si su intervención es en un tema contable específico, es necesario adaptarse a las nuevas normas que la empresa se encuentra implementando. Sin embargo, en razón a que los auditores son agentes ajenos a la empresa contratados bajo el régimen especial de prestación de servicios, no influyen considerablemente en el proceso de permeabilidad de las NIIF en la estructura organizacional.

4. Abogados: en cuanto al papel que cumplen los abogados, es necesario que estos tengan conocimiento acerca de la normatividad que rige las NiIf, qué normas las cobijan y legalmente qué ente las vigila, además de conocer en buena medida las NIIF debido a los procesos legales que podrían necesitar de dicho conocimiento.

Esta capacitación se llevará a cabo mediante:

1. Asesorías: los asesores tienen un alto conocimiento y experiencia en aspectos técnicos dependiendo del sector y las necesidades de la empresa. Esta brinda los requerimientos necesarios para la adopción de las NIIF la primera vez, así mismo 
las estrategias para llevar a cabo la asimilación del cambio, la revisión de políticas, la preparación de los estados financieros, el entrenamiento y el desarrollo de tareas sobre las NIIF aplicables a la compañía, y demás servicios.

2. Cursos en línea: este método se lleva a cabo cuando el personal no tiene el tiempo necesario para acudir a un curso presencial, y, cuando no se desea intervenir en las horas laborales, este se realiza a través de Internet utilizando una plataforma donde se le explica el proceso de implementación de las NIIF, utilizando videos, audio, gráficas y demás. Asimismo, se desarrollan actividades que evalúan el nivel de compresión o conocimiento que se tiene de la norma según el avance de cada sección.

3. Cursos de programación sistemática: como bien sabemos, para implementar esta norma la empresa deberá instalar un nuevo software, por tal motivo el personal deberá tener el conocimiento necesario para el buen manejo del nuevo sistema.

A continuación, se presenta el proceso previo de diagnóstico y planeación que debe llevarse a cabo antes de formular un proceso de trabajo de campo que conlleve a la creación de un cuadro de mando estratégico.

Trabajar con NIIF significa observar a la empresa y el entorno desde distintas perspectivas:

\section{Perspectiva de la PYME con relación a las NIIF}

¿Cómo elaborar una perspectiva dirigida a NIIF para PYME que aborde los obstáculos de la conversión desde el punto de vista organizacional?

- Distintas interpretaciones de las NiIf.

- Elevado costo de implementación.

- Aplicación rigurosa y consistente de las normas.

- Las distintas regulaciones que entran en conflicto.

- La dificultad para monitorear la aplicación de las normas.

- Oposición al proceso por parte de diversos grupos de interés.

- La dificultad de la medición del valor razonable en mercados no desarrollados.

- La falta de personal para la preparación y aseguramiento de la información (García, 2014).

Existiendo estos problemas y, a partir de la bibliografía revisada, se pueden entender las ventajas y desventajas que podría tener una PYME si incorpora las NIIF en su organización.

Sus ventajas son:

- Las normas NIIF para PYME son más simples y sencillas que las normas de NIIF completas.

- Mejora la calidad en cuanto a la presentación de información financiera.

- Ayuda a que las organizaciones cuenten con mayor transparencia y mayor confianza con sus inversionistas e instituciones financieras, etc.

- Mejora la comparabilidad de la información financiera de las PYME.

- Mayor facilidad y accesibilidad para obtener fuentes de financiamiento, tanto locales como extranjeras.

Sus desventajas son:

- No existe suficiente conocimiento de las NIIF para PYME en los países.

- No se ve la financiación por parte del Estado para la reeducación de los profesionales y profesores en los temas de normatividad internacional.

- Aumento en costos ya que la organización se verá obligada a capacitar a su personal y adquirir un nuevo software para el manejo de las NIIF.

- Resistencia por parte de los profesionales al incremento en la carga operativa, ya que se requiere mayor número de procedimientos al tener que llevar registros financieros y por separado los registros contables.

- Riesgo a que la información del contador y demás miembros de la organización no sean las adecuadas (Cedano et al., 2014).

Es importante mencionar que el papel del cliente en toda organización es muy importante, ya que uno de los fines de la empresa es satisfacer la necesidad del cliente; no obstante, cabe mencionar que el cliente es quien toma la decisión de comprar y el consumidor es quien se satisface. La idea de crear o de abrir nuevos horizontes hacia el exterior, donde se puedan adquirir nuevos clientes, suena exquisita para las organizaciones, porque no solo representa la imposición de marca, sino que también representa crecimiento financiero a la empresa, entre otros. No obstante, se sabe que es importante encantar al cliente prestando buenos servicios y productos de calidad para así lograr fidelizar y llamar la atención del cliente siempre hacia la empresa. 
Para lograr abrir nuevos mercados en el exterior hoy en día se está tratando de implementar las normas NIIF en las empresas con el objetivo de abrir nuevos horizontes en el exterior y adquirir nuevos clientes no solo nacionales, sino internacionales. Esta propuesta o idea para las empresas es muy interesante; no obstante, la implementación de esta norma influye en un cambio total de la organización desde el cargo más básico hasta los altos mandos con el riesgo de que esta estrategia de adquirir negocios internacionales falle; sin embargo, vale correr el riesgo al implementar esta norma, ya que para la empresa representa un logro en todas las áreas.

Las NIIF están relacionadas con los procesos internos clave de una organización, debido a que generan grandes cambios en la contabilización que se maneja. Por tal motivo, la empresa deberá acudir a un nuevo software que le facilite generar los estados financieros que son requeridos (tanto para las NIIF como para la DIAN). Igualmente se relaciona con la "perspectiva de aprendizaje y crecimiento", ya que dentro de la empresa se deberán realizar programas de formación al personal para que estos puedan adaptarse con mayor facilidad a los cambios contables, las operaciones igualmente tendrán un gran cambio ya que no se registrará la contabilidad de la manera en la que se realizaba anteriormente, y se darán grandes impactos en el reporte de los resultados. Esto puede aumentar costos y posiblemente se requiera de más herramientas para alcanzar los objetivos propuestos.

Tanto el personal contable, los directivos y todos los empleados de la organización deberán estar al tanto de los cambios que se pueden dar a la hora de la implementación de las NiIf. Se necesitará de comités de auditoría para estar verificando que la adaptación de las normas sea clara y precisa.

\section{Perspectiva del cliente}

La perspectiva del cliente nos indica el segmento del mercado que el negocio está dispuesto a atacar y que probablemente representan sus ingresos en el corto plazo. Esta perspectiva permite identificar los indicadores clave correspondientes a la satisfacción de los clientes, fidelidad y rentabilidad (Capítulo Iv, 2014).

La perspectiva del cliente mide factores tales como profesionalidad y buen trato, promociones, estabilidad de marca, experiencia de compra, satisfacción total, nuevas alternativas y calidad en el producto (Norton, 2001).

¿Dequémanerapodría afectarlaimplementación de las NIIF en la parte financiera de una organización?
Podemos ver que la parte financiera es importante en el cuadro de mando integral puesto que esta perspectiva nos puede ayudar a cumplir metas y objetivos propuestos tanto financiera como organizacionalmente (estrategias).

Esta perspectiva está estrechamente vinculada con implementación de las NIIF en las organizaciones, puesto que las NiIf están relacionadas con la parte financiera o contable. Por tal motivo, al implementarlas, puede afectar notoriamente la parte financiera de una organización, puesto que, al entrar en vigencia, debe haber unos cambios en los estados financieros, en la contabilidad y en la normatividad en general, cambiando así de mejor manera las diferentes metas o estrategias que se tienen previstas en una organización. Además de cambiar la manera de llevar la contabilidad, la aplicación de las NIIF nos puede llevar a una generación de costos un poco mayor, puesto que para llevar esto a cabo necesitamos implementar nuevas formas y tecnologías que pueden salir un poco costosas para la organización, pero que al final pueden ser de gran utilidad a la hora de hacer una implementación de las NiIf de forma clara y concisa para los diferentes organismos de la organización.

\section{Perspectiva de aprendizaje y crecimiento}

Esta perspectiva se basa en las necesidades en relación con las personas, la tecnología, los activos, las alianzas. Con esto se sustenta la excelencia de la organización. Además, se hacen preguntas tales como cuáles son las actividades o actitudes que debe tener el capital humano para hacer realidad los objetivos organizacionales. Además se deben tener en cuenta los siguientes ítems: número de nuevos productos, porcentaje en las ventas de nuevos productos, inversión en capacitación, número de habilidades estratégicas aprendidas $+\mathrm{D}$, porcentaje de las ventas, número de sugerencias de los empleados (Guerriero, 2009).

\section{Perspectiva interna}

Estos procesos son aquellos en los cuales una empresa debe ser excelente para satisfacer las necesidades de los clientes. Esta perspectiva incluye las actividades de una organización que están incorporadas en los procesos internos que forman la cadena de valor de la organización, tales como mejoras en la eficiencia, reducción de los costes unitarios, reducción de residuos, mejoras en la moral, aumento de la utilización de la 
capacidad de los empleados, aumento de la productividad, porcentaje de productos defectuosos y cantidad de residuos reciclados (Guerriero, 2009).

Son notorios los cambios que se producirán con la implementación de las NIIF, y la situación es preocupante, porque las empresas no tienen suficiente conocimiento del impacto y el proceso que implica la adopción de las NIIF. El principal problema que se da es en el tema del recurso humano, ya que, de los contadores registrados en Colombia, la mínima parte ha recibido capacitación; esto implica que estas personas deben prepararse lo antes posible o si no, las empresas van a incumplir en este proceso. Las NIIF implican cambios en la forma de llevar los libros, en los principios contables y en los informes que se generan ("Recurso humano, clave en el proceso de las normas NIIF", 2013). ¿Esto genera retos para la educación superior? El sistema educativo tiene la responsabilidad de modificar los pénsum de contaduría, por lo que están enseñando algo que posiblemente no se utilice; se necesita que estas introduzcan las NIIF para que los contadores futuros tengan conocimiento y no deban recurrir a capacitaciones ("El papel del contador en los procesos internacionales”, 2012).

Es el contador quien lleva el procedimiento a cabo, es el encargado de llevar los hechos y transacciones económicos dentro de la organización, cumpliendo así las normas y reglas propuestas para este fin. Las nuevas normas internacionales exigen, por tanto, a todos los profesionales (no solo a los contadores), conocer, estudiar, determinar su contenido, impacto, sus procesos y procedimientos para la manipulación de la información.

\section{Perspectiva financiera}

¿Cómo interpretar la perspectiva financiera de un cuadro de mando operativo, que incorpore las NIIF para PYME sobre las secciones que aplique a esta? Como bien se sabe Colombia se encuentra en un proceso de convergencia a las NIIF. Las NIIF para PYME se caracterizan por ser menos estrictas que las NIIF completas. Su estructura cuenta con 35 secciones.

De esas 35 secciones encontramos ocho secciones que nos hablan sobre los estados financieros y dos que abarcan los instrumentos financieros, los otros hablan de los activos, pasivos y patrimonio, ingresos, costos y gastos, entre otros.

Estados financieros:
- Sección 3: presentación de estados financieros.

- Sección 4: estado de situación financiera.

- Sección 5: estado de resultado integral y estado de resultados.

- Sección 6: estado de cambios en el patrimonio y estado de resultados y ganancias acumuladas.

- Sección 7: estado de flujo de efectivo.

- Sección 8: notas a los estados financieros.

- Sección 9: estados financieros consolidados y separados.

- Sección 10: políticas contables.

Instrumentos financieros:

- Sección 11: instrumentos financieros básicos.

- Sección 12: otros temas relacionados con los instrumentos financieros (IASB, 2009).

La perspectiva financiera del cuadro de mando integral del balance scorecard mantiene los indicadores financieros que son capaces de resumir las consecuencias económicas y el estado de una organización (Capítulo IV, 2014).

Es por lo anterior que la perspectiva financiera es prácticamente el culmen del ejercicio de la creación de mapas estratégicos, ya que, bajo sus indicadores, se medirá el impacto de las estrategias desarrolladas a partir de la perspectiva de aprendizaje. Los factores que evalúa la perspectiva financiera son: rentabilidad, crecimiento y desarrollo con sostenibilidad.

\section{Mapa estratégico}

Es importante para el trabajo tener en cuenta el modelo europeo de excelencia empresarial, ya que es reconocido como modelo $\mathrm{EFQM}$ y está patrocinado por la Fundación Europea para la Gestión de Calidad (EFQM) y la Comisión de la Unión Europea, base del Premio Europeo a la Calidad. Esto no llevará a tener un mejor control sobre el procedimiento y los beneficios que estos conllevan.

Los resultados han de mostrar tendencias positivas, compararse favorablemente con los objetivos propios y con los resultados de otras organizaciones, estar causados por los enfoques de los agentes y abarcar todas las áreas relevantes.

Lo que lleva a trabajar este proceso innovador desde un punto de competitividad a un nivel mayor, queriendo alcanzar la mejora de procesos desde la prospectiva, toma de decisiones y gerencia del talento humano. 


\section{Esquema del contenido del modelo EFQM}

Como se puede apreciar en la figura 1, la complejidad del modelo y la importancia que se puede dar a los aspectos de medición de una serie de variables que influyen a niveles de toda una empresa y como se pueden ejecutar en un cuadro de mandos.

El modelo EFQM de excelencia es un marco de trabajo no prescriptivo que tiene nueve criterios. Cinco de ellos son "agentes facilitadores" y cuatro son "facilitadores" (véase las tablas 1 y 2).

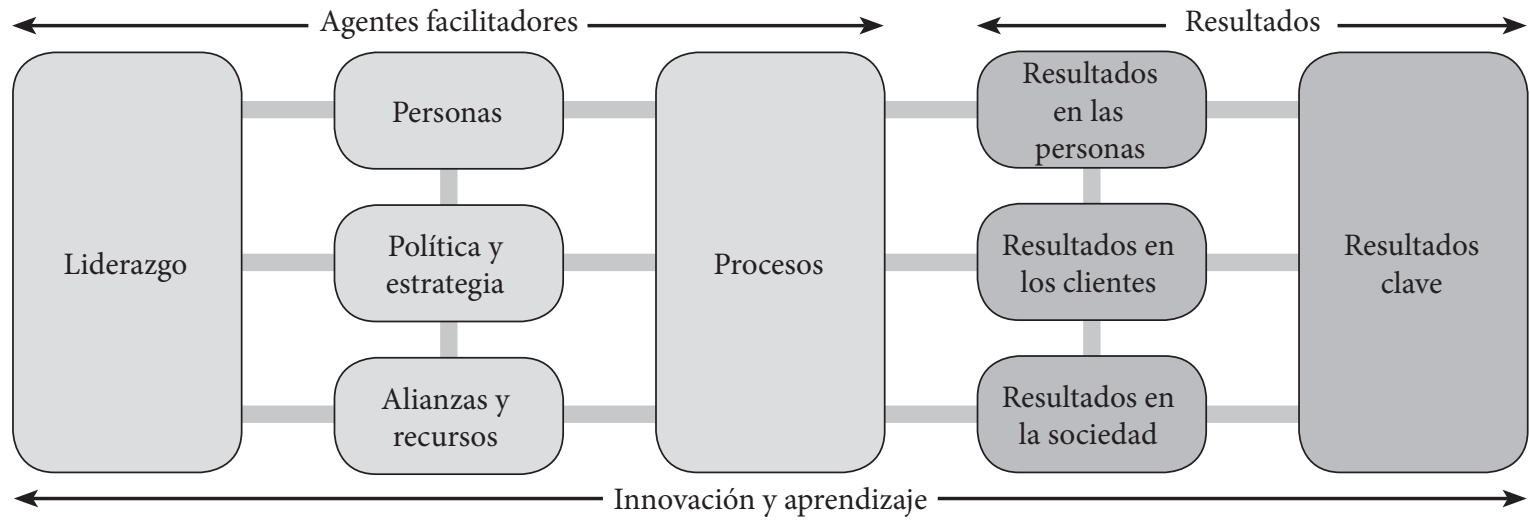

Figura 1. Modelo EFQM. Elaboración propia.

Tabla 1

Agentes facilitadores

\begin{tabular}{|l|l|}
\hline \multicolumn{2}{|l|}{ 1. Liderazgo } \\
\hline 1a & Directivos comprometidos con la gestión de calidad total \\
\hline 1b & $\begin{array}{l}\text { Los directivos dirigen activamente la mejora dentro de } \\
\text { la empresa y se implican con los clientes, proveedores y } \\
\text { otras organizaciones externas }\end{array}$ \\
\hline 2. Estrategia y planificación \\
\hline 2a & $\begin{array}{l}\text { Formular la estrategia y los planes basados en informa- } \\
\text { ción relevante y completa }\end{array}$ \\
\hline 2b & Comunicación e implantación de estrategia y planes \\
\hline 2c & Actualización y mejora de estrategia y planes \\
\hline 3. Gestión del personal \\
\hline 3a & Desarrollo y revisión de planes para el personal \\
\hline 3b & $\begin{array}{l}\text { Asegurar la implicación y asunción de responsabilidad } \\
\text { de los empleados }\end{array}$ \\
\hline 4. Alianzas y recursos \\
\hline 4a & Gestión de los recursos financieros \\
\hline 4b & Gestión de los recursos de información \\
\hline 4c & Gestión de los proveedores y materiales \\
\hline 4d & Gestión de otros recursos \\
\hline 5. Sistema de la calidad y procesos \\
\hline 5a & Orientación al cliente \\
\hline 5b & Gestión del sistema de la calidad \\
\hline 5c & $\begin{array}{l}\text { Gestión de los procesos clave para la generación de } \\
\text { productos y servicios }\end{array}$ \\
\hline $5 d$ & Gestión del proceso de mejora continua \\
\hline
\end{tabular}

Tabla 2

Resultados

\begin{tabular}{|l|l|}
\hline \multicolumn{2}{|l|}{ 6. Satisfacción del cliente } \\
\hline 6a & Resultados de la percepción del cliente \\
\hline & $\begin{array}{l}\text { La imagen general } \\
\text { Productos y servicios } \\
\text { Ventas y servicios posventa } \\
\text { Lealtad de los clientes }\end{array}$ \\
\hline 6b & Indicadores de satisfacción de los clientes \\
\hline 7. Satisfacción del personal \\
\hline 7a & Resultados de la percepción del personal \\
\hline & $\begin{array}{l}\text { Motivación } \\
\text { Satisfacción }\end{array}$ \\
\hline 7b & Indicadores de satisfacción del personal \\
\hline 8. Impacto en la sociedad \\
\hline 8a & Resultados del impacto en la comunidad \\
\hline & $\begin{array}{l}\text { Medio ambiente } \\
\text { Prevención de riesgos } \\
\text { Implicación activa en la comunidad }\end{array}$ \\
\hline 9. Resultados empresariales \\
\hline 9a & Resultados financieros \\
\hline 9b & Indicadores de la actuación de la empresa \\
\hline
\end{tabular}

Nota. Elaboración propia.

Nota. Elaboración propia. 
Estudios similares: en un estudio realizado por G. Frizelle (2002), del Institute for Manufacturing, University of Cambridge, se menciona que:

Ofrece datos interesantes más precisos. Explica los resultados de aplicar a 27 PYME (industriales y de servicios) del Reino Unido una herramienta sofisticada de planificación estratégica: Focus Strategy Management, cuya metodología y software fueron financiados por el British Department of Trade and Industry. Se ha analizado la herramienta y es ciertamente completa y avanzada, requiriendo de varias sesiones de trabajo. En el caso de las empresas del estudio contaron con consultor que les guiaba en el proceso. ¿Quélleva a las empresas PYME a embarcarse en un programa tan exigente (relativamente)? ¿Cuál es el desencadenante? En casi todos los casos se demostró que había una razón concreta (aparte de planificar para el futuro). Las razones son las que aparecen en el siguiente cuadro.

\section{Distribución de los tipos de eventos que accionan una revisión de la estrategia}

Tabla 3

Tipos de acción desencadenante con su respectiva frecuencia

\begin{tabular}{|l|c|}
\hline \multicolumn{1}{|c|}{ Acción desencadenante } & Frecuencia \\
\hline Redirección de pérdidas o pérdidas potenciales & 5 \\
\hline Nuevas oportunidades de expansión & 5 \\
\hline Reenfocar el negocio & 4 \\
\hline Llegada de un nuevo consejero delegado & 3 \\
\hline Presión exterior & 3 \\
\hline Designación de un nuevo equipo directivo & 2 \\
\hline Establecimiento del negocio & 1 \\
\hline Búsqueda de un socio & 1 \\
\hline Tamaño de la muestra & 27 \\
\hline
\end{tabular}

Nota. Tomado de Business Strategy: Do smes Face Special Problems? Proceedings of Stimulating Manufacturing Excellence in Small and Medium Enterprises (smesme) Conference 2001, 49, por G. Frizelle, 2002.

Este mapa estratégico lleva a una aplicación segura dentro de un marco que ya ha sido aplicado y permite develar que, de una forma organizada, se logran los objetivos establecidos al inicio de este proyecto y resolvemos la pregunta planteada sobre la adopción de las normas.

Aplicando las Pyme en el cuadro de mando, podría ser de esta forma que se engloban generalmente todas las áreas:

En relación con lo anterior se realizó un estudio similar correlacional a PYME nacionales buscando similitudes en los modelos presentados, este trabajo manejó cinco componentes o grupos que toda PYME posee, los cuales presentaron el siguiente resultado.

\section{Índice Kaiser-Meyer-Olkin}

El índice Kaiser-Meyer-Olkin (кмо) se utiliza para comparar las magnitudes de los coeficientes de correlación parcial, de forma que cuanto más pequeño sea su valor, mayor será el valor de los coeficientes de correlación parciales ij (p) r y, en consecuencia, menos apropiado es realizar un análisis factorial.

Kaiser, Meyer y Olkin, para realizar un análisis factorial, proponen que si en el test su resultado KMO es mayor o igual a 0,9 , es muy bueno; si es mayor o igual a 0,8 , es notable; si es mayor o igual a 0,7 , será mediano; si es mayor o igual a 0,6 , será bajo, y si es menor a 0,5 , muy bajo.

Tabla 4

KMO, prueba de Bartlett y varianza total explicada

\begin{tabular}{|ll|l|}
\hline \multicolumn{2}{|c|}{ Notas } \\
\hline Recursos & Tiempo de procesador & $00: 00: 00: 50$ \\
& Tiempo transcurrido & 00:00:00:52 \\
& Memoria máxima & $74020(72.285 \mathrm{~K})$ bytes \\
& necesaria & \\
\hline
\end{tabular}

\{conjunto_de_datos\} C: /users/Tishiba/Dropbox/UMNG/Investigación/Proyecto NIIF/Base_PYMMES_NIIF.sav

\begin{tabular}{|l|l|l|}
\hline \multicolumn{2}{|c|}{ KMO y prueba de Bartlett } \\
\hline $\begin{array}{l}\text { Medida de adecuación muestral } \\
\text { de Kaiser-Meyer-Olkin }\end{array}$ & .900 \\
\hline $\begin{array}{l}\text { Prueba de electrici- } \\
\text { dad de Bartlett }\end{array}$ & Chi-cuadrado aproximado & 2.125 .729 \\
\hline & gl & 300 \\
\hline & Sig. & .000 \\
\hline
\end{tabular}


Tabla 4

кMо, prueba de Bartlett y varianza total explicada (continuación)

\begin{tabular}{|c|c|c|c|c|c|}
\hline \multicolumn{6}{|c|}{ Varianza total explicada } \\
\hline \multirow{2}{*}{ Componente } & \multicolumn{3}{|c|}{ Autovalores iniciales } & \multicolumn{2}{|c|}{$\begin{array}{l}\text { Suma de las saturaciones al } \\
\text { cuadrado de la rotación }\end{array}$} \\
\hline & Total & $\%$ de la varianza & $\%$ Acumulado & Total & $\%$ de la varianza \\
\hline 1 & 10.329 & 41.316 & 41.316 & 4.004 & 16.014 \\
\hline 2 & 2.495 & 9.982 & 51.298 & 3.830 & 15.321 \\
\hline 3 & 1.287 & 5.147 & 56.445 & 3.397 & 13.589 \\
\hline 4 & 1.184 & 4.736 & 61.181 & 3.066 & 12.265 \\
\hline 5 & 1.031 & 4.124 & 65.305 & 2.029 & 8.115 \\
\hline 6 & .878 & 3.513 & 68.818 & & \\
\hline 7 & .759 & 3.037 & 71.855 & & \\
\hline 8 & .732 & 2.929 & 74.784 & & \\
\hline 9 & .714 & 2.857 & 77.642 & & \\
\hline 10 & .647 & 2.587 & 80.228 & & \\
\hline
\end{tabular}

Este índice compara la magnitud de los coeficientes de correlación observados con la magnitud de los coeficientes de correlación parcial. Los valores pequeños del estadístico KMO indican que las correlaciones entre los pares de variables no pueden explicarse por otras variables y que tal vez el análisis factorial no sea adecuado. Por lo general, es deseable un valor mayor a 0,5 ; para nuestro resultado, el кмо fue 0,900 , lo cual es un dato representativo y deseable, coeficiente muy cercano a la unidad que es lo más aconsejado, lo que indicaría una perfecta adecuación de los datos a un modelo de análisis factorial.

En nuestro método Káiser, se puede comprobar, y así lo hemos señalado, que, a partir de la componente número cinco, el autovalor comienza a ser inferior a la unidad, aunque en este componente todavía mantiene un valor alto. Además, el porcentaje de la varianza explicada acumulada asciende al 65,305\% de la varianza total, por lo que se puede considerar que este puede ser un valor lo suficientemente alto para estimar que cuatro es un número de factores suficiente. Los datos adjuntos corresponden a la medición de cinco componentes, cuyas variables medidas y resultados obtenidos fueron los que se muestra en la tabla 5 .

\section{Tabla 5}

Resultados de la medición de cinco componentes

\begin{tabular}{|l|c|c|}
\hline \multirow{4}{*}{$\begin{array}{l}\text { Componente } \\
\text { Varianza }\end{array}$} & 1 & 41,316 \\
\cline { 2 - 3 } & 2 & 9,982 \\
\cline { 2 - 3 } & 3 & 5,147 \\
\cline { 2 - 3 } & 4 & 4,736 \\
\cline { 2 - 3 } & 5 & 4,124 \\
\hline
\end{tabular}

Nota. Resultados del sPss.

A su vez, los autovalores expresan la cantidad de la varianza total que está explicada por cada factor y por los porcentajes de varianza explicada, asociados a cada factor; estos últimos se obtienen dividiendo su correspondiente autovalor por la suma de los autovalores.

\section{Prueba de esfericidad de Bartlett}

La prueba de esfericidad de Bartlett contrasta la hipótesis nula de que la matriz de correlaciones observada es en realidad una matriz identidad. Asumiendo que los datos provienen de una distribución normal 
multivariante, el estadístico de Bartlett se distribuye aproximadamente según el modelo de probabilidad chi-cuadrado y es una transformación del determinante de la matriz de correlaciones. Si el nivel crítico (sig.) es mayor que 0,05 , no se podrá rechazar la hipótesis nula de esfericidad y, consecuentemente, no se podrá asegurar que el modelo factorial sea adecuado para explicar los datos.

En síntesis, mediante la prueba de esfericidad de Bartlett, se puede asegurar que si el nivel crítico es superior a 0,05 , entonces no se podrá rechazar la hipótesis nula de esfericidad. Como podemos comprobar en nuestro análisis, la significación es perfecta, ya que obtiene el valor 0,000 . Por esto, se puede rechazar dicha hipótesis nula, considerando el ajuste de las variables mediante el análisis factorial idóneo, y se acepta la hipótesis alterna.

A continuación, se analizarán los componentes en función de las variables iniciales que incorporan:

Componente 1. Este componente engloba al conjunto de atributos que se concentran en la encuesta como pertenecientes al grupo "crecimiento de ganancia, ingresos e inversión" (CGI), ya que se considera que dan la sensación y hacen que este factor de resultados sea el más representativo y seguro. Este será la componente que denominamos "factor CGI", y que explica por sí sola nada menos que el $41,316 \%$, casi la mitad de la varianza total (tabla 4), ocupando destacadamente el primer lugar frente al resto de componentes que se van a presentar.

Componente 2. Contiene cinco variables iniciales que son consideradas como "secretos y propiedad intelectual" (SPI) y que resulta lógico que presenten información redundante. Llamaremos a esta componente el factor de sPI y explica un 9,982\% de la varianza total (tabla 6).

Componente 3. Incluye las características en lo relacionado con "Costos, mercadeo, políticas y descuentos" (CMPD) que manejan las PYME de forma idónea para el consumidor. Se ha denominado a esta componente el "factor CMPD garantía de empresa", explicando por sí sola el 5,147\% del total de la varianza (tabla 7).

Tabla 6

Matriz de componentes rotados: componente 1 y 2

\begin{tabular}{|c|c|c|c|c|c|}
\hline & \multicolumn{5}{|c|}{ Componente } \\
\hline & 1 & 2 & 3 & 4 & 5 \\
\hline $\begin{array}{l}\text { El crecimiento de nuestras ganancias es mucho mayor que el crecimiento de las } \\
\text { ganancias en nuestra industria }\end{array}$ & 736 & & & & \\
\hline $\begin{array}{l}\text { Nuestros inversionistas apoyan el uso de instrumentos financieros de cobertura, } \\
\text { bonos, préstamos y compra de acciones para la administración del negocio }\end{array}$ & 723 & & & & \\
\hline $\begin{array}{l}\text { Tenemos una medición fiable de ingresos y diferenciación sobre el origen de } \\
\text { las ganancias derivada de la gestión del negocio y de las ganancias transitorias } \\
\text { obtenidas por títulos valores o préstamos a futuro }\end{array}$ & 694 & & & & \\
\hline $\begin{array}{l}\text { Tenemos un plan de inversión en propiedades, ya sea en el corto, mediano o largo } \\
\text { plazo }\end{array}$ & 694 & & & & \\
\hline $\begin{array}{l}\text { La empresa tiene una proyección de inversión en subsidiarias en el largo plazo } \\
\text { para sacarle provecho al goodwill del negocio de la industria }\end{array}$ & 682 & & & & \\
\hline $\begin{array}{l}\text { Nuestra organización está más orientada a la estandarización en la presentación } \\
\text { de los estados financieros y estados contables básicos }\end{array}$ & 633 & & & & \\
\hline $\begin{array}{l}\text { Identificamos y numeramos nuestros secretos empresariales, know how, patentes, } \\
\text { investigaciones y modelos de negocios, y los introducimos en los informes }\end{array}$ & & -779 & & & \\
\hline $\begin{array}{l}\text { Reconocemos el valor de nuestra marca, su registro, estrategia e identidad, y nos } \\
\text { adaptamos a las normas internacionales para incluirlas en los balances y resulta- } \\
\text { dos de la empresa }\end{array}$ & & -761 & & & \\
\hline $\begin{array}{l}\text { Utilizamos las herramientas y prácticas comunes de nuestro sector, como plata- } \\
\text { formas, sistemas, software y aplicaciones para mejorar niveles de competitividad } \\
\text { del personal y de la empresa, en el ámbito local e internacional, y le damos a la } \\
\text { empresa sus certificaciones }\end{array}$ & & -712 & & & \\
\hline
\end{tabular}

Nota. Elaboración propia. 
Tabla 7

Matriz de componentes rotados: componente 3

\begin{tabular}{|c|c|c|c|c|c|}
\hline & \multicolumn{5}{|c|}{ Componente } \\
\hline & 1 & 2 & 3 & 4 & 5 \\
\hline Hacemos provisión de indemnización por años de servicios u otros beneficios al personal. & & -647 & & & \\
\hline $\begin{array}{l}\text { Hacemos operaciones en moneda extranjera dentro de un plan de conversación del negocio } \\
\text { en el extranjero. }\end{array}$ & & -643 & & & \\
\hline $\begin{array}{l}\text { Los componentes de los costos de ventas como adquisición, producción, costos directos e } \\
\text { indirectos, etc., están sopesados en relación con el beneficio que se entrega al cliente. }\end{array}$ & & & 783 & & \\
\hline En nuestra empresa, evidenciamos una economía de escala que beneficia al cliente. & & & 694 & & \\
\hline $\begin{array}{l}\text { Nuestras estrategias de mercadeo están orientadas a hacer crecer todo el mercado del sector, } \\
\text { en lugar de crecer la participación en el mercado existente. }\end{array}$ & & & 686 & & \\
\hline $\begin{array}{l}\text { Nuestra empresa tiene una política clara de discriminación y reducción de costos que no } \\
\text { disminuyan en el valor entregado al cliente, pero que sí generan mayor rentabilidad. }\end{array}$ & & & 633 & & \\
\hline $\begin{array}{l}\text { Las rebajas en descuentos comerciales por compran anticipadas son de alguna manera } \\
\text { trasladados en beneficios de valor a nuestro cliente final. }\end{array}$ & & & 579 & & \\
\hline $\begin{array}{l}\text { ¿El centro corporativo tiene muy bien diferenciadas cuales son las ganancias financiadas, } \\
\text { obtenidas con instrumentos financieros y acciones? }\end{array}$ & & & & & \\
\hline $\begin{array}{l}\text { Tenemos ejemplos claros del pasado y plenamente divulgados sobre cómo ganaremos todo } \\
\text { cuando trabajamos unidos como empresa. }\end{array}$ & & & & 749 & \\
\hline $\begin{array}{l}\text { Fortalecemos permanentemente un sentido compartido de propósitos y valores que sostie- } \\
\text { nen la cultura de la empresa y estimulamos el desempeño tanto individual como colectivo. }\end{array}$ & & & & 690 & \\
\hline
\end{tabular}

Nota. Elaboración propia.

Tabla 8

Matriz de componentes rotados: componente 4 y 5

\begin{tabular}{|c|c|c|c|c|c|}
\hline & \multicolumn{5}{|c|}{ Componente } \\
\hline & 1 & 2 & 3 & 4 & 5 \\
\hline Tenemos unas claras normas de conducta que definen nuestra identidad como empresa. & & & & 663 & \\
\hline $\begin{array}{l}\text { Los límites de nuestro negocio no están determinados por la cantidad de producto vendido, } \\
\text { sino por el valor del beneficio que generamos al cliente. }\end{array}$ & & & & 604 & \\
\hline $\begin{array}{l}\text { Existe una tendencia a la estandarización de los costos activados por mantenimientos, inspec- } \\
\text { ciones generales, por defectos y reemplazos, para reducir fluctuaciones en las cotizaciones. }\end{array}$ & & & & & \\
\hline $\begin{array}{l}\text { Para el desarrollo de nuevos productos y servicios de nuestra compañía, involucramos } \\
\text { previamente las necesidades del cliente. }\end{array}$ & & & & & 797 \\
\hline $\begin{array}{l}\text { Cada vez que emprendemos un proyecto nuevo en nuestra compañía, involucramos a los } \\
\text { clientes para incrementar su beneficio. }\end{array}$ & & & & & 711 \\
\hline $\begin{array}{l}\text { En nuestra empresa, invertimos en investigación y desarrollo, independientemente de las } \\
\text { fluctuaciones del sector y nuestro mercado. }\end{array}$ & & & & & \\
\hline
\end{tabular}

Método de extracción: análisis de componentes principales.

Método de rotación: normalización Varimax con Kaiser

a. La rotación ha convergido en siete iteraciones

Nota. Elaboración propia. 
Tabla 9

Matriz de transformación de los componentes

\begin{tabular}{|c|c|c|c|c|c|}
\hline Componente & $\mathbf{1}$ & $\mathbf{2}$ & $\mathbf{3}$ & $\mathbf{4}$ & $\mathbf{5}$ \\
\hline 1 & 527 & 511 & 455 & -444 & 238 \\
\hline 2 & -421 & -444 & 529 & -172 & 560 \\
\hline 3 & 622 & -351 & 187 & -641 & 213 \\
\hline 4 & -393 & 592 & 373 & -597 & 2 \\
\hline 5 & 26 & -262 & 582 & -84 & -765 \\
\hline
\end{tabular}

Método de extracción: análisis de componentes principales.

Método de rotación: normalización Varimax con Kaiser.

Nota. Elaboración propia.

Componente 4. Cuatro variables conforman este factor: misión, cultura y visión de la PYME (MVC). Se cita este componente como el factor de MVC, ya que este factor recoge las características más políticas de la empresa, explicando el $4,736 \%$ de la varianza total (tabla 8 y 9 ).

Componente 5. Cuatro variables conforman este factor: innovación e investigaciones en las PYME (II). Se denomina a esta componente el "factor de II", ya que este factor recoge las características sobre las políticas de la empresa, explicando el $4,124 \%$ de la varianza total (tabla 5).

Una vez que se han obtenido las componentes, se pueden lograr y definir las puntuaciones factoriales mediante una estimación para cada uno de los sujetos en cada factor extraído, para así valorar qué situación tiene ese sujeto frente a estas nuevas variables que se han construido a partir de la reducción de las variables iniciales introducidas en el análisis.

Cuando se utiliza el método de extracción de factores utilizando las componentes principales, las puntuaciones obtenidas se calculan a partir de la solución factorial. Esta solución es ortogonal, por lo que las puntuaciones también lo son; estas permiten separar los efectos de cada uno de los factores sobre la variable de interés.

En síntesis, es importante este diseño porque se puede estimar cada efecto principal e interacción de forma independiente. La ortogonalidad garantiza que el efecto de un factor o interacción pueda estimarse de manera independiente del efecto de cualquier otro factor o interacción presente en el modelo.

Es importante entender el principio de la corresponsabilidad y el compromiso de cada individuo con respecto al trabajo de la empresa, en el que se tenga claro qué hace en el lugar de trabajo, cuáles son sus obligaciones, responsabilidades y derecho; que pueda comprender qué es la planificación personal, y la planificación empresarial. Como se pudo observar con el ejercicio realizado, cada área tiene una responsabilidad de llegar a ser altamente competitiva ya que cada una es vocera y responsable de las inversiones realizadas en las empresas para los procesos innovadores y de gestión, que conllevan a un nivel tecnológico superior y poseen un plan estratégico.

En la formación y el desarrollo de los trabajadores y directivos, el área de recursos humanos debe destacar la existencia de sanas relaciones entre el equipo directivo y la del resto de sus empleados. A mayor formación del equipo directivo, mayor es la cualificación requerida al personal colaborador.

Este trabajo deja de lado las posibilidades de crear y continuar innovando temas tales como los factores que inciden en la consolidación de plantilla de las PYME, la creación de nuevas variables de medición de formación de empleados y directivos, la aplicación de la PYME y su crecimiento en los contextos local y nacional, etc.

\section{Cuadro de mando}

Es importante tomar como base el cuadro de mando de Kaplan y Norton (figura 2).

En relación con la Business Intelligence, Kaplan y Norton (1996) anuncian algo muy importante en el cuadro de mando, lo cual rescato para su ejecución y tener un concepto claro y específico. Señalan:

Es de suma importancia rescatar que se denomina Cuadro de Mando Integral (CMI) a aquella herramienta de gestión empresarial que es útil para medir la evolución de la actividad de una compañía y sus resultados, desde un punto de vista estratégico y con 


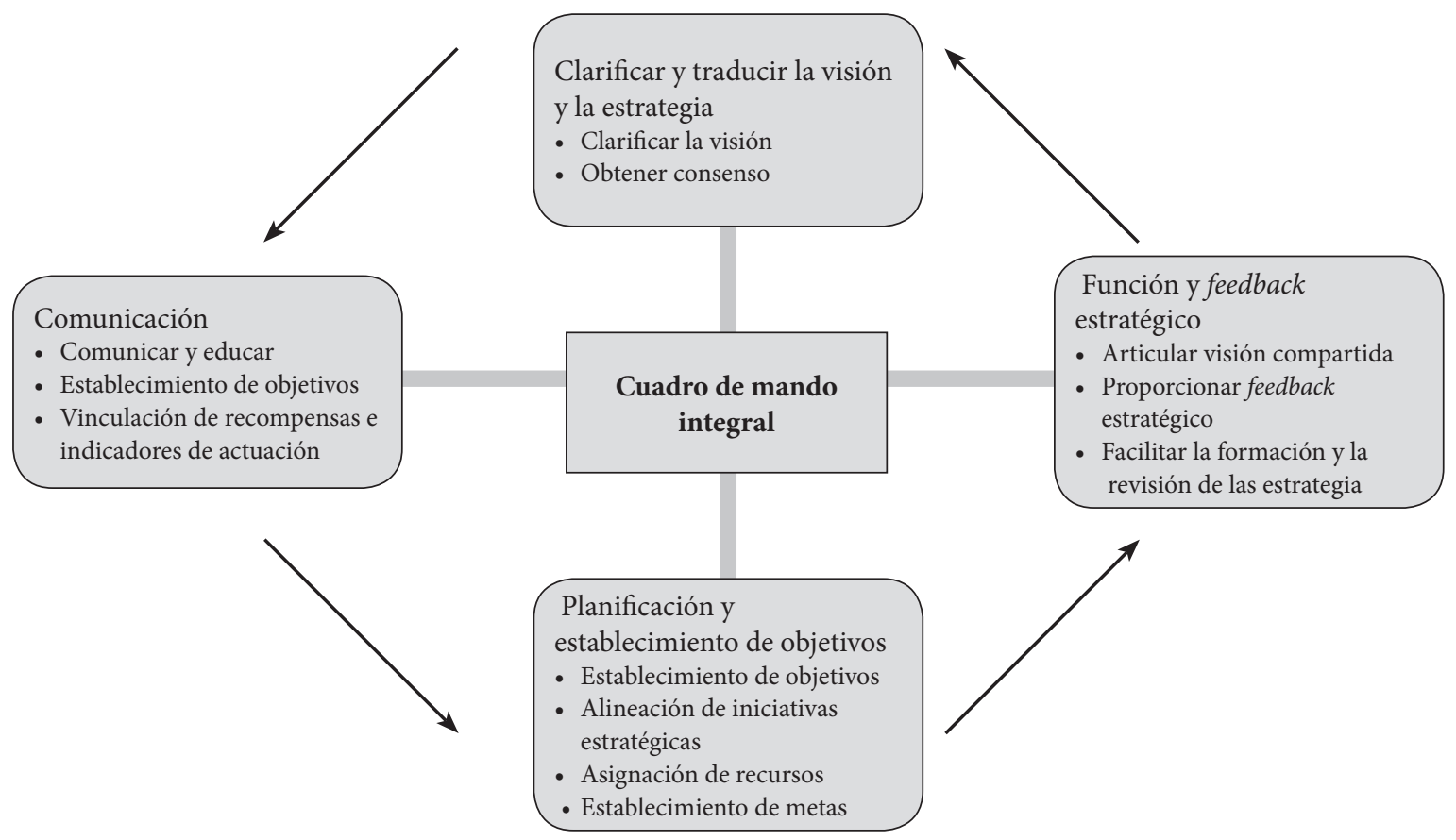

Figura 2. Tomada de "Using the balanced scorecard as a strategic management system", por R. S. Kaplan y D. P. Norton, 1996, en Harvard business review, 85(7-8), p. 150.

Tabla 10

Resultado del cuadro de mando y su plan estratégico

\begin{tabular}{|c|c|c|}
\hline \multirow[t]{2}{*}{ Objetivos estratégicos } & \multicolumn{2}{|c|}{ Indicadores estratégicos } \\
\hline & Indicadores de efecto & Indicadores de causa \\
\hline $\begin{array}{l}\text { Financieros: } \\
\text { 1. Mejorar los rendimientos } \\
\text { 2. Ampliar diversidad de ingresos } \\
\text { 3. Reducir la estructura de coste }\end{array}$ & $\begin{array}{l}\text { 1. Rendimiento sobre la inversión } \\
\text { 2. Crecimiento de ingresos } \\
\text { 3. Cambio de coste de servicio de depósito }\end{array}$ & Variedad de ingresos \\
\hline $\begin{array}{l}\text { Clientes: } \\
\text { Aumentar satisfacción del cliente y aumen- } \\
\text { tar satisfacción posventa }\end{array}$ & $\begin{array}{l}\text { Cuota de segmento } \\
\text { Retención de clientes }\end{array}$ & $\begin{array}{l}\text { Profundidad de la relación "Encuesta de } \\
\text { satisfacción" }\end{array}$ \\
\hline $\begin{array}{l}\text { Interno: } \\
\text { Comprensión a los clientes } \\
\text { Crear e innovar productos } \\
\text { Reducir problemas operativos } \\
\text { Un servicio sensible }\end{array}$ & $\begin{array}{l}\text { Cambio de la combinación de canales, tasa } \\
\text { de errores del nuevo servicio, tiempo de } \\
\text { cumplimiento de una solicitud }\end{array}$ & Ciclo de desarrollo horas dedicas al cliente \\
\hline $\begin{array}{l}\text { Formación: } \\
\text { Desarrollo de las habilidades estratégicas, } \\
\text { proporcionar información estratégica, ali- } \\
\text { near los objetivos personales a incentivos }\end{array}$ & $\begin{array}{l}\text { Satisfacción del empleado ingresos por } \\
\text { empleado }\end{array}$ & $\begin{array}{l}\text { Ratio de cobertura del trabajo } \\
\text { Información estratégica } \\
\text { Ratio de disponibilidad de la información } \\
\text { estratégica } \\
\text { Porcentaje de alineación de metas personales }\end{array}$ \\
\hline
\end{tabular}

Nota. Elaboración propia. 
una perspectiva general. Gerentes y altos cargos la emplean por su valor al contribuir de forma eficaz en la visión empresarial, a medio y largo plazo.

Saber establecer y comunicar la estrategia corporativa para alinear los recursos y las personas en una dirección determinada no es tarea sencilla, y un Cuadro de Mando Integral resulta de gran ayuda para lograrlo. A través de sus indicadores de control, financieros y no financieros, se obtiene información periódica para un mejor seguimiento en el cumplimiento de los objetivos establecidos previamente, y una visión clara del desarrollo de la estrategia. Así, la toma de decisiones resulta más sencilla y certera, y se pueden corregir las desviaciones a tiempo.

El uso y aplicación de un Cuadro de Mando Integral es no solo posible, sino también aconsejable para empresas medianas y pequeñas. Su efectividad no depende del tamaño de la compañía, así que tanto las grandes organizaciones como las PyME pueden aprovecharse de sus enormes beneficios.

Es de aclarar que existe un software que maneja en forma integrada la gestión del desempeño de la estrategia al enlazarla con la gestión de los proyectos requeridos para llevarla a cabo y con la documentación de los procesos, la gestión de compromisos y de los riesgos, y la toma de decisiones, lo cual hace que sea aún más fácil adquirir una herramienta que se pueda adaptar a las condiciones de cada necesidad.

En la tabla 10, se observa el resultado esperado del cuadro de mando y un plan estratégico para ejecutarlo.

Por otra parte, el cuadro de mando para control es muy completo y vale resaltarlo en este análisis, ya que no se trata de inventar (existe ya hace décadas), sino de utilizar aquellas herramientas que nos permitan facilitar los procesos a nivel gerencial y desde allí coordinar todas y cada una de las áreas de la compañía para estar a un nivel superior en cuanto a los mercados.

\section{Conclusiones}

En la elaboración del trabajo, se han analizado los procedimientos de la NIIF para las PYME adoptando dicha norma para preparar estados financieros en un cuadro de mando, lo cual lleva a la conclusión de que es una herramienta de gran utilidad para cualquier compañía. El cambio contable que la adopción de la norma implica contempla criterios simplificados.

Mediante el proceso y desarrollo del trabajo, se concluye que el beneficio de la aplicación de las NIIF, especialmente cuando se realiza la comparación de la aplicación de la sección 9, un alto impacto y a su vez es una de las secciones más importantes y relevantes en este trabajo, por el alto volumen de cuentas por cobrar. Por ende, el impacto de conversión se hace favorable para la empresa que se tomó como ejemplo. Por otra parte, al revisar otra de las secciones, con un un impacto más bajo, se encuentra en la sección 1, ya que no es significativo a nivel de cifras en los procesos. Lo que sí se resalta es que el contar con información útil, real y oportuna puede facilitar la toma de decisiones en un momento determinado.

Por otra parte, para completar lo enunciado, cabe resaltar o mejor rescatar las NIC como impacto NIIF para las PYME, ya que tienen una gran relevancia en este trabajo y se destacan las NIC 12, 16 y 36 ; con un impacto medio las NIC 7 y 20 , y con menor influencia las NIC 2, 19, 37, 38, 39.

Por consiguiente, el CMI es un medio que permite estimular y obtener beneficio, teniendo la capacidad de combinar recursos y talentos del personal, llevando a una motivación intrínseca desde el área de recursos humanos y que se verá reflejada en la organización y a su vez en la aceptación de los clientes que son los que al final estimulan la mejora de resultados económicos y los procesos.

Finalmente, se corroboró que CMI es correlacional en la implementación del modelo en las Pyme; los resultados fueron más que probabilísticos.

\section{Referencias}

Business Intelligence. (2014). Recuperado de http://www. lantares.com/blog/bid/331346/Cuadro-de-MandoIntegral-Todo-lo-que-Debes-Saber.

Cedano, S., et al. (2014). NIIF para PYME. Bogotá.

El papel del contador en los procesos internacionales. (2012, noviembre 6). Portafolio. Recuperado de http://www. portafolio.co/economia/el-papel-del-contador-los-procesos-internacionales

Filion, L. J., Cisneros-Martínez, L. F. y Mejía-Morelos, J. H. (2011). Administración de PYME: emprender, dirigir y desarrollar empresas. México: Pearson Educación.

Frizelle, G. (2002). Business Strategy: Do smes Face Special Problems? Proceedings of Stimulating Manufacturing Excellence in Small and Medium Enterprises (sMESME) Conference 2001, 49.

García, S., Sanabria, P. y Ospina, D. (2013). Balanced Scorecard for Entrepreneurial Strategic Marketing in Colombia. Global Journal of Business Research, 7(4), 47-59. Disponible en https://ssrn.com/abstract=2239212. 
García, S. y Ogliastri, J. G. (2014). Cuadro de mando integrado con las Normas Internacionales de Información Financiera para pyme (Proyecto de investigación). Bogotá: Universidad Militar Nueva Granada y la Universidad La Gran Colombia.

Guerriero, D. (2009). Indicadores para cada perspectiva. Recuperado de http://inghenia.com/wordpress/2009/08/29/ balanced-scorecard-indicadores-para-cada-perspectiva/

Consejo de Normas Internacionales de Contabilidad. (IASB). (2009). Norma internacional de Información Financiera para Pequeñas y Medianas entidades (NIIF para PYMES). Londres: IASB. Disponible en https://incp.org.co/Site/2014/ mailing/spatia/ES_IFRS_for_SMEs_Standard.pdf

IFRs Colombia. (2014). 7 consejos para prepararse para las NIIF. Colombia: Karulin.
Kaplan, R. S. y Norton, D. P. (1996). Using the Balanced Scorecard as a Strategic Management System. Harvard Business Review, 85(7-8), 150.

Norton, D. (2001). Cómo utilizar el cuadro de mando integral. Barcelona: Gestión 2000.

Capítulo Iv. (2014). Recuperado de http://tesis.uson.mx/digital/tesis/docs/19072/Capitulo4.pdf

Recurso humano, clave en el proceso de las normas NiIf. (2013, 8 de octubre). La República. Recuperado de http://www.larepublica.co/finanzas/recurso-humano-clave-en-el-proceso-de-las-normas-niif_68321.

Salazar, C. A. (2013). Tendencias de la auditoría internacional. Armenia: Editorial Universitaria de Colombia.

Viveros, M. L. (2014). Normas Internacionales de Información Financiera Hacia la implementación empresarial. Bogotá. 